\title{
Lesión traumática del tronco cerebral diagnosticada por resonancia magnética. Reconsideración epidemiológica y pronóstica
}

\author{
J. Aguas; R. Begué* y J. Díez*
}

Servicio de Neurocirugía. Institut de diagnostic per l'imatge*. Hospital Universitari Arnau de Vilanova. Lleida

\section{Resumen}

Introducción y Objetivos. La TAC craneal permite la evaluación quirúrgica urgente de un TCE, pero no supone una evaluación completa de las lesiones encefálicas producidas. La resonancia magnética $(\mathrm{RM})$ puede complementar la evaluación del TCE, especialmente a nivel de tronco cerebral. Utilizando la secuencia FLAIR pretendemos obtener una estimación de la frecuencia de lesión primaria traumática de tronco cerebral.

Material y métodos. Se presenta una serie prospectiva de 30 casos con TCE moderado o grave (GCS $\leq 13)$ a los que se les realizó RM en un intervalo menor a dos semanas tras el traumatismo. En la serie se incluyeron exclusivamente pacientes jóvenes (entre 16 y 40 años), con objeto de excluir lesiones previas de tipo isquémico no relacionadas con el traumatismo. Quedaron fuera los pacientes con cirugía craneal para excluir lesiones yatrogénicas.

En base a estudios previos, se utilizó la secuencia FLAIR (Fluid Attenuated Inversion Recovery 8000/ 120/ T. Inversion $2200 \mathrm{mseg}$ ) para la detección óptima de lesiones de tronco cerebral.

Resultados. En un $26,6 \%$ de los casos se apreciaron lesiones en tronco cerebral confirmadas por dos radiólogos independientes. De ellas, seis casos correspondían a lesiones hiperintensas compatibles con lesión axonal difusa y dos casos a lesión hemorrágica. La supervivencia de la serie fue del $100 \%$, si bien este dato está sesgado por la selección exclusiva de pacientes que en un plazo inferior a dos semanas habían salido de cuidados intensivos. En cuatro casos pudo establecerse una relación directa entre lesión y focalidad neurológica. En el resto, la lesión fue relacionada con trastornos inespecíficos del nivel de consciencia.

Conclusiones. Consideramos que la RM en secuencia FLAIR nos permite visualizar un tipo de lesión traumática de tronco cerebral (posiblemente lesión

Recibido: 26-01-04. Aceptado: 16-04-04 axonal) que presenta mayor frecuencia y menor gravedad pronóstica que aquellas otras descritas clásicamente en estudios realizados mediante TAC.

PALABRAS CLAVE

Tronco cerebral. Traumatismo craneal. Resonancia magnética. Secuencia FLAIR. Lesión axonal difusa.

Brainstem injury diagnosed by MRI. An epidemiologic and prognostic reappraisal

Summary

Introduction and Objectives. CT-scan allows emergency surgical evaluation of head injury lesions, but does not offer a comprehensive diagnosis of the resulting brain injuries. Magnetic Resonance Imaging (MRI) can complete the evaluation of head injury, particularly in the brain stem. We attempted to estimate the frequency of traumatic primary brain stem injuries by using the FLAIR (Fluid Attenuated Inversion Recovery) sequence.

Material and Methods. Thirty patients with moderate or severe head injury (GCS $\leq 13)$ underwent a MRI study during the first two weeks after trauma. In order to exclude old patients with previous ischemic lesions unrelated to the head trauma, only young patients (16-40 years-old) were included. Patients with cranial surgery were also eliminated from the study. Based on previous studies, the FLAIR (8000/120/T. Inversion $2200 \mathrm{mseg}$ ) sequence was selected.

Results. Brain stem injuries were detected in $\mathbf{2 6 . 6 \%}$ of the patients; this was confirmed by two independent radiologists. Six patients had hyperintense lesions compatible with diffuse axonal damage, and two others

Abreviaturas. DP: densidad protónica. FLAIR: fluid attenuated inversion recovery. GCS: Glasgow coma scale. LAD: lesión axonal difusa. LED: lesión encefálica difusa. RM: resonancia magnética. TAC: tomografía axial computarizada. TCE: traumatismo craneoencefálico. 
showed hemorrhagic lesions. These findings were directly related to a specific neurological deficit in four patients; while in the remaining, unspecific consciousness disturbances were noted.

Conclusions. We believe that the FLAIR sequence demonstrate a type of traumatic brain stem injury (probably corresponding to diffuse axonal injury) that is more frequent and less severe in terms of prognosis than those classically described in previous CT scan studies.

\section{KEY WORDS}

Brainstem. Head injury. Magnetic Resonance imaging. FLAIR sequence. Diffuse axonal injury.

\section{Introducción}

La definición y epidemiología de la lesión traumática de tronco es un tema controvertido dentro del estudio del traumatismo craneoencefálico. De hecho, deberíamos cuestionarnos si se trata de una única entidad o de varias. En la literatura, los datos son contradictorios $\mathrm{y}$, mientras unos autores la consideran una lesión de carácter primario relativamente frecuente, otros cuestionan su existencia como lesión primaria ${ }^{3,6,11,14}$

Ello no es de extrañar, ya que los métodos para su diagnóstico hasta la última década, o bien no han sido demasiado sensibles (TAC craneal y potenciales evocados), o bien suponían un sesgo metodológico (series autópsicas).

En el ámbito clínico, esta falta de definición ha llevado al establecimiento de conceptos que deberían ser replanteados: La lesión traumática de tronco ¿es exclusiva del TCE grave o también puede aparecer en el moderado o leve? ¿Implica siempre mal pronóstico? ¿Es una lesión axonal o hemorrágica?

La utilización de la RM podía aportar una valiosa información al respecto, pero los primeros estudios realizados mostraron contradicciones por discrepancias metodológicas. Era preciso seleccionar la secuencia más adecuada para su detección y es en 1997 cuando queda demostrada la superioridad de la secuencia FLAIR para la detección de lesiones traumáticas no hemorrágicas ${ }^{1}$.

Hemos considerado de interés estudiar de forma prospectiva los hallazgos obtenidos, mediante esta secuencia, en un grupo de traumáticos craneales para obtener una estimación de la frecuencia de las lesiones traumáticas de tronco en nuestro medio.

\section{Material y métodos}

Durante un periodo de 32 meses, comprendido entre Septiembre de 2000 y Mayo de 2003, se estudió una serie prospectiva de traumatismos craneales moderados y graves, (GCS $<13$ en el momento de su llegada a urgencias) que cumplieran los siguientes criterios:

a) Adulto sin antecedentes patológicos, con edad comprendida entre 16-40 años. Con ello, se pretendía excluir patología vascular propia de edades más avanzadas, así como cualquier otra patología neurológica que pudiera interferir en la interpretación de las imágenes.

b) Ausencia de intervención quirúrgica intracraneal (a excepción de monitorización de presión intracraneal) que pudiera determinar lesiones iatrogénicas añadidas.

c) Ausencia de inestabilidad hemodinámica en cuidados intensivos que determinase posible lesión secundaria.

d) Posibilidad de realización de resonancia magnética en un plazo inferior a dos semanas tras el traumatismo. En nuestro medio, esta condición implica el alta de cuidados intensivos durante el mencionado tiempo y por tanto selecciona un grupo de traumatismos craneales con evolución favorable.

Se obtuvieron mediante esta selección los 30 pacientes que conforman la presente serie.

Según la situación clínica del paciente, su estancia durante las dos primeras semanas tuvo lugar en cuidados intensivos o planta hospitalaria. El GCS medio de la serie fue de 9.7 puntos. La media de edad fue de 23.7 años. Dados los criterios de inclusión, la evolución de la serie fue muy favorable con una supervivencia del $100 \%$ y un Glasgow outcome score (GOS) a los 6 meses de 4,66.

El estudio mediante técnicas de imagen consistió en la TAC craneal de urgencias (siguiendo la clasificación de Marshall $^{10}, 14$ casos fueron considerados grado I, 12 casos grado II y 4 casos grado III), completado con el correspondiente control durante la primera semana. La resonancia magnética se realizó entre la primera y segunda semana.

Todos los pacientes fueron estudiados con una RM de 1,5 Tesla (Philips Gyroscan Intera) empleando una bobina de cuadratura específica de cráneo. Las imágenes de RM fueron evaluadas por dos radiólogos independientemente. El protocolo de estudio incluyó las siguientes secuencias: Sagital SE T1 (TR/TE 480/13), matriz de 256 x 256, campo de visión de $250 \mathrm{~mm}$ con grosor de corte de $5 \mathrm{~mm}$ y $1 \mathrm{~mm}$ de distancia entre cortes. Axial SE T1 (TR/TE 539/15), matriz de 256 x 256, campo de visión de $220 \mathrm{~mm}$ con grosor de corte de $6 \mathrm{~mm}$ y $0,6 \mathrm{~mm}$ de distancia entre cortes. Axial SE en DP (Densidad Protónica) y T2 (TR/TE 2087/ 20 2087/90), matriz de i56 x 256, campo de visión de 230 $\mathrm{mm}$ con grosor de corte de $6 \mathrm{~mm}$ y $0,6 \mathrm{~mm}$ de distancia entre cortes. Axial y Coronal FLAIR (Fluid Attenuated Inversión Recovery) (TR/TE/TI 8.000/120/2.200), matriz de 256 x 512, campo de visión de $220 \mathrm{~mm}$ con grosor de corte de $6 \mathrm{~mm}$ y $1,2 \mathrm{~mm}$ de distancia entre cortes.

\section{Resultados}

Las lesiones intracraneales diagnosticadas mediante 
Tabla 1

Relación de hallazgos diagnósticos mediante TAC y las distintas secuencias de RM.

\begin{tabular}{|c|c|c|c|c|c|}
\hline$N^{3}$ & $\mathrm{CCS}$ & TAC & KM-T1 & $\mathrm{RM}-\mathrm{T2}$ & RM-ILAIR \\
\hline 1 & 13 & HSA & HSA & TISA & IISA \\
\hline 2 & 13 & F. hemorrag. & Cont. lobar & Cont. lobar & Conl. lobar \\
\hline .3 & 10 & F. hemincrady. & Conll. lathar & Cont. latkis & Comt. lobier \\
\hline 1 & 7 & Conl. lobar & Conl. lolkar & Cont. lotsur & Cont. lobiur \\
\hline 5 & 13 & Conl. lokar & Conil. lotkar & 2 Conl- hobar & 2 Cont. lobiar \\
\hline 20 & 6 & F. hemorrag & Cont. lobur & Cont. lobrur & Cont. lobar \\
\hline 13 & 11 & 4Conil lukar & ZCont lobiar & 4Cont lobar & 4Cont lobar \\
\hline 18 & 3 & Cont lobar + trunce & Cont lobir - tronco & Cont lohar+ tronco & Cont lobar + tronco \\
\hline 29 & 6 & Conl lobart tronco & Cint lobir - muncu & Cont lobar + tronce & Cont lobar + tronco \\
\hline 25 & 8 & Conil lothrar & Cinal kitair (O) & Cont.lubirr (2) & Cont lobar (2) \\
\hline 6 & 10 & & Cont. lobiar & Contlub + g.banales & Cont. lobar + g.basales \\
\hline 14 & 13 & . & & C'ont lobiar & C'ont lobar \\
\hline 21 & 13 & 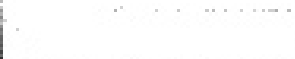 & - & Cont lobar & C'ont lobar \\
\hline 7 & 10 & 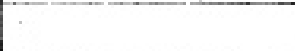 & - & C'ont suboortical & C'ont. subcortical \\
\hline 8 & 9 & - & $\cdots \cdot \cdot \cdot$ & C. callos & g.basales + C. calloso + tronco \\
\hline 28 & 11 & - & & C. dalloso + tronco & Cont lob + C. calloso + tronco \\
\hline 23 & 13 & - & & S. calloso & C. calloso \\
\hline 27 & 6 & - & & & g.basales + tronco \\
\hline 9 & 13 & - & & - & C. calloso + tronco \\
\hline 10 & 7 & - & 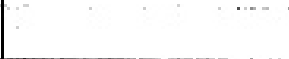 & - & C. calloso I tronco \\
\hline 12 & 13 & - & - & $\because$ & KCont lobar + C.calloso \\
\hline 16 & 14 & - & - & - & Cont lobar \\
\hline 22 & 6 & Netumoencefalo & - & $\cdots$ & Cont. lobar \\
\hline 15 & 10 & H5A & - & & Cont lobar+ suboort tronco \\
\hline 79 & 6 & HSA & - & $\cdot$ & C. calloso \\
\hline 30 & 1.3 & Cont. loluar & - & - & Cont. lobar \\
\hline 17 & 6 & Cont + h. subdural & - & & Cont lobar \\
\hline 24 & 12 & Cont. puntiforme & - & & - \\
\hline 11 & 10 & - & - & & - \\
\hline 26 & 6 & - & & - & - \\
\hline
\end{tabular}

HSA: hemorragia subaracnoidea. F. Hemorrag.: foco hemorrágico. Cont lobar: contusión lobar. G gasales: lesión en ganglios basales. C Calloso: lesión en cuerpo calloso. tronco: lesión en tronco cerebral. -: sin hallazgos.

técnicas de imagen se detallan en la tabla 1. En la columna correspondiente a TAC se incluyen los hallazgos obtenidos, ya sea en la exploración de urgencias o en el posterior control. En las tres siguientes columnas se distingue entre secuencias T1, T2 y FLAIR. Cabe señalar que la secuencia potenciada en $\mathrm{T} 1$ ofrece un rendimiento diagnóstico inferior al TAC (11 casos con hallazgos diagnósticos mediante RM en T1 frente a 16 con TAC). La RM en T2 permite un ligero aumento en hallazgos diagnósticos con respecto al TAC, (17 frente a 16). Eso sí, ignorando lesiones visualizadas en 
TAC y aportando nuevos diagnósticos en otros casos.

Pero la técnica que marca la diferencia por su mayor sensibilidad, tanto frente al TAC como a las otras secuencias RM, es la secuencia FLAIR. De hecho, obtiene diagnóstico de lesiones encefálicas, en general, en 28 de los 30 casos (93.3\%); lo que queda muy lejos de cualquiera de las otras técnicas juntas o por separado.

Centrándonos en las lesiones de tronco cerebral, hemos de considerar como patrón oro la RM en secuencia FLAIR, dada su mayor capacidad para la detección de lesiones encefálicas. Con dicha secuencia, hemos obtenido diagnóstico de lesión de tronco cerebral en ocho de los 30 casos $(26,3 \%)$. Mediante otras pruebas o secuencias los resultados han sido más pobres: dos casos mediante TAC, dos casos mediante RM en T1 y tres casos mediante RM en T2. Todos los casos diagnosticados mediante TAC, T1 o T2 también los fueron mediante secuencia FLAIR.

En cuanto a la características específicas de las imágenes obtenidas con dicha secuencia distinguimos seis lesiones hiperintensas no hemorrágicas y laterales, una lesión hiperintensa hemorrágica y lateral y una lesión hipointensa, hemorrágica y medial.

Al considerar las características clínicas del subgrupo de pacientes con lesión de tronco se aprecia un GCS medio a su ingreso de 8,1 (frente al 9,7 en el conjunto de la serie). En cuanto al resultado se obtuvo un GOS medio de 4,25 a los seis meses, mejor de lo habitualmente esperado para las lesiones de tronco, pero peor que el general de la serie 4,66.

En cuatro, de los ocho casos, pudo establecerse una relación directa entre lesión y focalidad neurológica; en el resto, la lesión fue relacionada con trastornos inespecíficos del nivel de consciencia.

Al cabo de seis meses se realizó una RM de control, apreciándose el carácter transitorio de estas lesiones en siete de los ocho casos.

\section{Casos ilustrativos}

\section{Caso 15}

Paciente de 18 años que presenta TCE como consecuencia de un accidente de tráfico (pasajera en vehículo). A su llegada a urgencias, presenta agitación psicomotriz y disminución del nivel de consciencia (GCS:10). En la TAC craneal se aprecia discreta hemorragia subaracnoidea postraumática. Tras una breve estancia preventiva en UCI, pasa a planta donde mejora el nivel de consciencia, pero persiste el estado de agitación durante una semana. La RM en secuencia FLAIR pone de manifiesto lesión parietal subcortical izquierda y lesión de tronco cerebral a nivel mesencefálico postero lateral derecho (Fig 1). La evolución clínica de la paciente fue satisfactoria y la RM en secuencia FLAIR a los 6 meses normal.

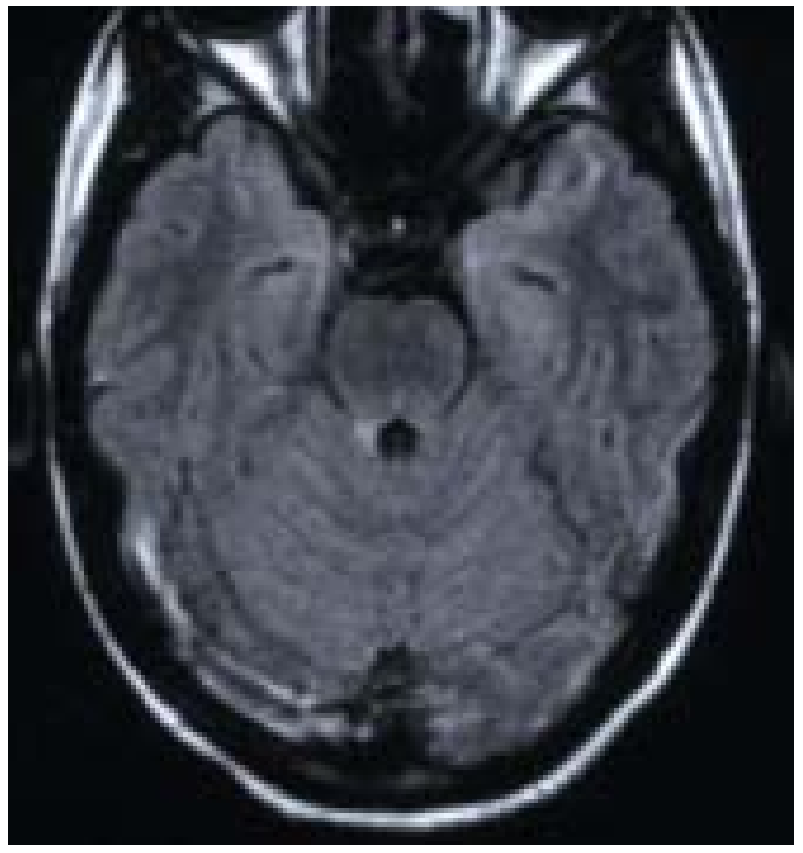

Figura 1. Caso 15. RM en secuencia FLAIR, corte axial. Lesión de tronco cerebral a nivel mesencefálico posterolateral derecho.

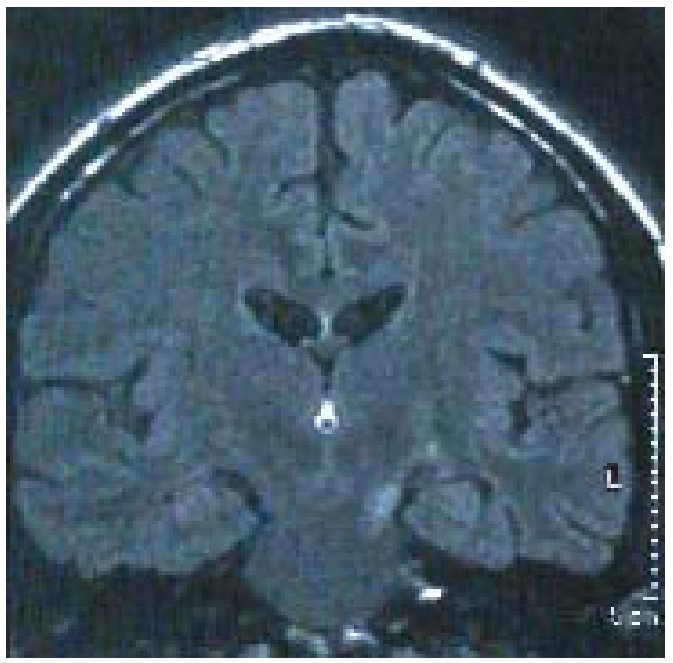

Figura 2. Caso 27. RM en secuencia FLAIR, corte coronal. Lesión a nivel de tronco cerebral (pedúnculo cerebral izquierdo).

\section{Caso 27}

Paciente de 28 años que presenta TCE como consecuencia de accidente laboral (vuelco de vehículo industrial). A su llegada a urgencias, presenta importante deterioro del nivel de consciencia GCS: 6 y hemiplejia derecha. Es intubado y en ventilación asistida se realiza TAC craneal que resulta ser normal. Pasa a UCI donde se retira sedación y es extubado al cabo de $24 \mathrm{~h}$. Se aprecia importante recuperación del nivel de consciencia GCS: 13 
pero persistencia de hemiplejia. El TAC de control tampoco aporta hallazgos diagnósticos. Se remite a planta donde se aprecia hemiparesia grave $1 / 5$. Se realiza RM al cabo de una semana tras el traumatismo. En ella se aprecia lesión a nivel de tronco cerebral (pedúnculo cerebral izquierdo) que explica el cuadro clínico (Fig 2). Al cabo de 6 meses, se produjo una notable recuperación de la hemiparesia 4/5 y normalización de las imágenes obtenidas en secuencia FLAIR.

\section{Discusión}

\section{Desarrollo histórico del concepto de lesión de tronco}

Las primeras referencias bibliográficas, que tratan de estudiar las características y frecuencia de la lesión primaria de tronco cerebral, datan de principios de los 70 , antes incluso del empleo sistemático del TAC craneal en el estudio del traumatismo craneal. La serie autópsica de Crompton (1971), basada en 106 casos $^{3}$, define una ubicación característica de lesión primaria a nivel posterolateral y una frecuencia considerable del 30\%. Esta idea es radicalmente rebatida por Mitchell y Adams en su articulo de 1973 "Primary focal impact damage to the brainstem in head injury, does it exist?", donde defienden la idea de un cuadro de lesión difusa de la sustancia blanca.

El uso generalizado del TAC craneal como prueba diagnóstica fundamental $\mathrm{y}$, por consiguiente, la baja frecuencia de lesiones de tronco diagnosticadas mediante esta técnica, inclinó la balanza a favor de Mitchell y Adams: La lesión de tronco fue relegada a un papel marginal tanto en el ámbito clínico como académico.

Unicamente los patólogos insistían en sus series autópsicas. Britt publicaba una serie de 162 casos en 1980, en la que la lesión primaria de tronco aparecía en $25 \%$ de los $\operatorname{casos}^{2}$. En contrapartida, ese mismo año se publica un importante trabajo radiológico en el que Tsai et al, mediante una serie de 1600 exploraciones de TAC craneal $^{14}$ en traumatismos, cifra la frecuencia de lesión primaria de tronco en 1,2\%. Obviamente esta serie sólo mostraba lesiones hemorrágicas, pero volvía a resaltar el carácter marginal de la lesión de tronco; además, se asociaba a la idea de mal pronóstico, pues así sucedía en la mayoría de estas hemorragias mesencefálicas o pontinas.

Faltaba una respuesta desde el área de las técnicas de imagen, pero utilizando la RM, no el TAC, como método diagnóstico. Se produjo en 1989, cuando una serie de Gentry et al (70 casos) describe la presencia de lesiones, no hemorrágicas, sino caracterizadas por hiperseñal en T2. Obtienen una frecuencia de lesión primaria en el 24,3\% de los $\operatorname{casos}^{6}$. No obstante, sus resultados no son apoyados por el prestigioso grupo de Jenkins y Teasdale que en una serie similar ${ }^{7}$ conceden mayor importancia a las lesiones subcorticales y restan protagonismo a las lesiones de tronco. También en esta línea, Levin et al publican su serie de 94 traumatismos con estudio $\mathrm{RM}^{9}$. En ella postulan la abundancia de lesiones subcorticales y profundas de carácter primario por lesión axonal difusa, incluso lo relacionan con la disminución inicial del nivel de consciencia, pero no consideran el tronco cerebral como una localización específica de estudio.

El desarrollo en 1991 del Traumatic Coma Data Bank tuvo una gran repercusión en el ámbito neuroquirúrgico. De hecho, a partir de él aparece la clasificación de Marshall $^{10}$ del TCE en 6 grupos claramente definidos según sus imágenes en TAC craneal. No es nuestra intención restar mérito al gran avance unificador que ha supuesto este trabajo. En general, es la clasificación de referencia en nuestro ámbito y tiene valor pronóstico con respecto a la posible evolución de la presión intracraneal ${ }^{12}$. Sin embargo, creemos pertinente realizar dos matizaciones: 1) Ignora la RM y sus posibles hallazgos. Dentro del grupo LED I de Marshall (ausencia de lesiones en TAC) cabría distinguir subgrupos según existieran lesiones mediante RM. 2) La idea de lesión encefálica difusa (LED) es brillante como concepto abstracto, pero engloba lesiones focales que en determinados casos y localizaciones pueden tener un significado singular.

Este contexto de principios de los noventa tampoco era el adecuado para el estudio de las lesiones traumáticas de tronco. La situación empezará a cambiar en 1997 cuando Ashikaga establece la superioridad de la secuencia FLAIR para la detección de lesión axonal difusa ${ }^{1}$. A partir de allí, varios trabajos vuelven a interesarse por la frecuencia de la lesión de tronco en series de traumatismos: Firsching realiza sus estudios durante la estancia del paciente en $\mathrm{UCI}^{4,5}$. En una serie de 102 pacientes, con GCS de entrada 6 y mortalidad del $27 \%$, encuentra lesiones de tronco en $64 \%$ de los casos. En 2000, Shibata distingue dos tipos de lesiones según la posterior evolución ${ }^{13}$ : las dorsolaterales, de curso más benigno, y las dorsales profundas, de peor evolución. Por otra parte, Kampfl utiliza el diagnostico de lesión de tronco en RM como elemento pronóstico negativo para la recuperación de TCE en estado vegetativo ${ }^{8}$. Obtiene una elevada frecuencia de lesiones dorsolaterales en su serie ( $74 \%$ en pacientes vegetativos persistentes y $26 \%$ en no persistentes).

El momento actual se caracteriza por una mayor disponibilidad de la RM y sus diferentes secuencias. Probablemente las llamadas lesiones encefálicas difusas en TAC puedan ser matizables mediante RM; especialmente en aquellos casos en los que exista cierta incongruencia clínico radiológica. Con ello serán diagnosticadas con mayor frecuencia lesiones de tronco y otras estructuras neuroaxiales (como cuerpo calloso) y se redefinirá su frecuencia, naturaleza y pronóstico. 


\section{Nuestros hallazgos}

Nuestra serie selecciona metodológicamente un grupo de pacientes con TCE moderado o grave que evoluciona bien (alta de UCI en menos de dos semanas). En ello se diferencia de la serie de Firsching (que realiza la RM durante la estancia en $\mathrm{UCI}^{4}$ ) y de la serie de $\mathrm{Kampfl}$ (que se centra en TCE grave con estado vegetativo a las 6-8 semanas tras el traumatismo ${ }^{8}$ ). Por tanto, los hallazgos de nuestro trabajo habrán de ser valorados en su propio contexto. Ahora bien, un elemento común y llamativo con las citadas series es que la frecuencia de lesiones de tronco deja de ser marginal y se convierte en un porcentaje considerable (26,6\% de nuestra serie).

Una característica original de nuestra serie es que rompe con otra idea, relativamente extendida, en relación con el pronóstico infausto de estas lesiones. Es posible tener una lesión de tronco objetivada en secuencia FLAIR y tener un resultado evolutivo favorable. Así lo justifica nuestra supervivencia del $100 \%$ y GOS de 4,25 en el subgrupo con lesiones de tronco.

Ello no contradice el axioma sostenido por la bibliografía $^{8,9,14,15}$ de que la lesión de tronco empeora el pronostico; de hecho el GOS global de nuestra serie $(4,66)$ fue superior al resultante en el subgrupo con lesiones de tronco.

Surge una cuestión básica: ¿Qué tipo de entidad o entidades anatomopatológicas estamos detectando en nuestra serie o en las series recientes que utilizan la secuencia FLAIR?

No nos referimos a las lesiones hemorrágicas (poco frecuentes e inespecíficas en FLAIR), sino a las otras más frecuentes (6+1 en nuestra serie), hiperintensas en FLAIR $\mathrm{y}$ ausentes en TAC.

Ashikaga las considera como focos de lesión axonal difusa (LAD) ${ }^{1}$, aunque no existe evidencia anatomopatológica de ello. Se trataría de un incremento de contenido de agua en las proximidades de aquellas zonas con mayor disrupción axonal. Resulta paradójico que la lesión axonal difusa sea focal, pero así lo muestran las imágenes obtenidas.

Por otra parte, en nuestra serie y en otras revisadas se aprecia coincidencia de lesiones en tronco cerebral y en cuerpo calloso, lo que podría hacer suponer cierto componente de acción sobre el neuroeje en su mecanismo de producción. Wedekind et al lo atribuyen al modelo fisiopatológico centrípeto ${ }^{15}$, según el cual la mayor gravedad del traumatismo estaría directamente relacionada con la mayor profundidad de las lesiones axonales.

La ubicación más frecuente en nuestra serie ha sido dorsolateral, concretamente a nivel de pedúnculos cerebelosos ( 5 de los 8 casos, una de ellas con pequeño componente hemorrágico), y demostraron tener un curso benigno como anticipaba el trabajo de Shibata ${ }^{13}$. También encontramos 2 lesiones laterales a nivel de pedúnculo cerebral, donde lo más notorio fue su clara correlación con una focalidad neurológica.

Completó la serie una lesión hemorrágica ventromedial que evolucionó satisfactoriamente, pero dejó como secuela una lesión permanente en la reactividad pupilar. No encontramos ninguna lesión dorsal profunda, las de peor pronóstico según Shibata.

Como conclusión final, señalaremos la utilidad que hemos encontrado en la secuencia FLAIR, no solo para replantear la incidencia y el pronóstico de estas lesiones, sino como elemento práctico que permitió una valoración completa del cuadro clínico presentado por el paciente.

\section{Bibliografía}

1. Ashikaga, R., Araki, Y., Ishida, O.: MRI of head injury using FLAIR. Neuroradiology 1997; 39: 239-242.

2. Britt, R., Herrick, M., Mason, R., et al.: Traumatic lesions of the pontomedullary junction. Neurosurgery. 1980; 6: 623-631.

3). Crompton, M.R.: Brainstem lesions due to closed head injury. Lancet 1971; 1 (7701): 669-673.

4. Firsching, R., Woischneck, D., Diedrich, M., et al.: Early magnetic resonance imaging of brainstem lesions after severe head injury. J Neurosurg 1998 ; 89: 707-712.

5. Firsching, R., Woischneck, D., Klein, S., et al.: Classifícation of severe head injury based on magnetic resonance imaging. Acta Neurochir (Wien) 2001; 143: 263-271.

6. Gentry, L.R., Godersky, J.C., Thompson, B.H.: Traumatic brain stem injury: MR imaging. Radiology 1989;171: 177-187.

7. Jenkins, A., Teasdale, G., Hadley, M.: Brain lesions detected by magnetic resonance imaging in mild severe head injuries. Lancet 1986, 2 (8504): 445-446.

8. Kampf, A., Schmutzhard, E. Franz, G.: Prediction of recovery from post-traumatic vegetative state with cerebral magnetic-resonance ímaging. Lancet 1998; 351 (9118):17631767.

9. Levin, H.S., Williams, D., Crofford, M.: Relationship of depth of brain lesions to consciousness and outcome after closed head injury. J Neurosurg 1988; 69: 861-866.

10. Marshall, L.F., Marshall, S.B., Klauber, M.R., et al.: A new classifícation of head injury based on computed tomography. J Neurosurg 1991 ;75 (suppl): 14-20.

11. Mitchell, D., Adams, H.: Primary focal impact damage to the brainstem in blunt head injuries does it exist? The Lancet 1973 August 4: 215-218.

12. Poca, M.A., Sahuquillo, J., Baguena, M., et al.: Incidence of intracranial hipertensión atter severe head injury: a prospective study using the Traumatic Coma Data Bank classifícation. Acta Neurochir Suppl (Wien) 1998; 71: 27-30.

13. Shibata, Y., Matsumura, A., Meguro, K., et al.: Diffe- 
rentiation of mechanism and prognosis of traumatic brainstem lesions detected by magnetic resonance imaging in the acute stage. Clin Neurol Neurosurg 2000 ; 102: 124-128.

14. Tsai, F., Teal, J., Quinn, M.F., et al.: CT of brainstem injury. AJR 1980;134: 717-723.

15. Wedekind, C., Hesselmann, V., Lippert-Grüner, M.: Trauma to the pontomesencephalic brainstem - a major clue to the prognosis of severe traumatic brain injury. Br J Neurosurgery 2002; 16: 256-260.
Aguas J.; Begué R.; Díez J.: Lesión traumática de tronco cerebral diagnosticada por resonancia magnética. Reconsideración epidemiológica y pronóstica. Neurocirugía 2005; 16: $14-20$

Correspondencia postal: Dr. Jesús Aguas Valiente. Servicio de Neurocirugía. Hospital Universitari Arnau de Vilanova de Lleida. Avenida Rovira Roure, 80. 25198 Lleida 